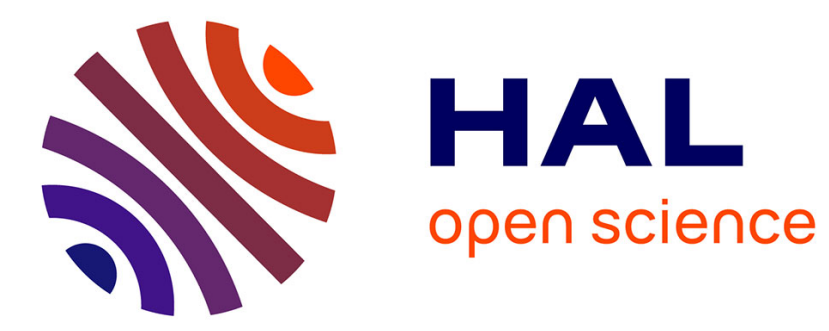

\title{
A mechanism accounting for independence on starting length of tension increase in ramp stretches of active skeletal muscle at short half-sarcomere lengths
}

\author{
Olaf Till, Tobias Siebert, Reinhard Blickhan
}

\section{- To cite this version:}

Olaf Till, Tobias Siebert, Reinhard Blickhan. A mechanism accounting for independence on starting length of tension increase in ramp stretches of active skeletal muscle at short half-sarcomere lengths. Journal of Theoretical Biology, 2010, 266 (1), pp.117. 10.1016/j.jtbi.2010.06.021 • hal-00613136

\author{
HAL Id: hal-00613136 \\ https://hal.science/hal-00613136
}

Submitted on 3 Aug 2011

HAL is a multi-disciplinary open access archive for the deposit and dissemination of scientific research documents, whether they are published or not. The documents may come from teaching and research institutions in France or abroad, or from public or private research centers.
L'archive ouverte pluridisciplinaire $\mathbf{H A L}$, est destinée au dépôt et à la diffusion de documents scientifiques de niveau recherche, publiés ou non, émanant des établissements d'enseignement et de recherche français ou étrangers, des laboratoires publics ou privés. 


\section{Author's Accepted Manuscript}

A mechanism accounting for independence on starting length of tension increase in ramp stretches of active skeletal muscle at short half-sarcomere lengths

Olaf Till, Tobias Siebert, Reinhard Blickhan

PII: $\quad$ S0022-5193(10)00311-5

DOI: $\quad$ doi:10.1016/j.jtbi.2010.06.021

Reference: $\quad$ YJTBI6041

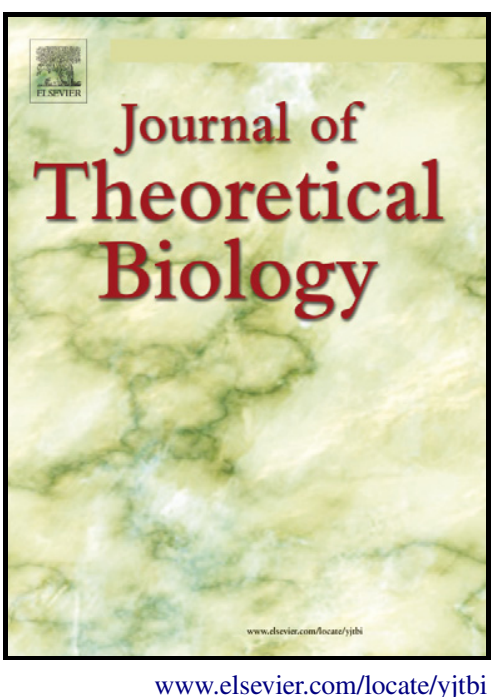

To appear in: $\quad$ Journal of Theoretical Biology

Received date: 18 January 2010

Revised date: 25 May 2010

Accepted date: 14 June 2010

Cite this article as: Olaf Till, Tobias Siebert and Reinhard Blickhan, A mechanism accounting for independence on starting length of tension increase in ramp stretches of active skeletal muscle at short half-sarcomere lengths, Journal of Theoretical Biology, doi:10.1016/j.jtbi.2010.06.021

This is a PDF file of an unedited manuscript that has been accepted for publication. As a service to our customers we are providing this early version of the manuscript. The manuscript will undergo copyediting, typesetting, and review of the resulting galley proof before it is published in its final citable form. Please note that during the production process errors may be discovered which could affect the content, and all legal disclaimers that apply to the journal pertain. 


\title{
A mechanism accounting for a tension increase independent of independence on starting length of tension increase in eccentric ramp stretches of active skeletal muscle eontractionsat short half-sarcomere lengths.
}

Olaf Till*, Tobias Siebert, Reinhard Blickhan

Division of Motion Science, Institute of Sport Science, Friedrich Schiller University, Seidelstraße 20, D-07749 Jena, Germany

\begin{abstract}
Based on previous experimental results of a length-independent inerease of tension with extension distance in ecentric independence on starting length of the tension gradient in constant-velocity contractions of a skeletal muscle stretches of active skeletal muscle at muscle lengths including the ascending limb and the plateau of the tension-length relation, a possible physiological mechanism for determining the tension increase in these contractions lengthening active muscle is discussed. Considering the sliding filament theory, it is suggested that the tension-length relation of a half-sarcomere in eccentric - lengthening contractions is different from that in isometric contractions. The assumed mechanism predicts, among others, that the thick filament retains its shortened length in eccentric lengthening contractions starting from a half-sarcomere length where this filament is compressed. An example model is implemented and checked with simulations.
\end{abstract}

Keywords: Skeletal muscle, Eccentric contraction, Muscle model, Simulation

*Corresponding author. Tel.: +49 36419 45713; fax: +4936419 45702. E-mail address: olaf.till@uni-jena.de (O. Till) 


\section{Introduction}

In physiologic motion contracting skeletal muscles may not only be shortened (= concentric contraction) or retain a constant length (= isometric contraction), but may also be lengthened (= eccentric contraction). It is well known that in maximally activated skeletal muscles, tension in isometric contractions is enhanced after a period of lengthening (Abbott and Aubert, 1952). This effect of contraction "history" is called "force enhancement". Suggested physiological explanations are altered crossbridge-cycling characteristics (e.g. Mehta and Herzog, 2008), enhanced elastic stiffness (e.g. Pinniger et al., 2006), andrapid lengthening of some half-sarcomeres to lengths at which they generate only passive tension ("sarcomere popping", Morgan et al. (2000) = no experimental evidence as yet, Telley et al. (2006)), and just heterogeneity of sarcomere lengths-lengthening velocity without "popping" (Telley et al., 2003, 2006). It is also known that tension can already be "enhanced" during lengthening in an extension-distance dependent way, in the sense that there is a tension increase with lengthening at constant velocity, even though purely isometric contractions at the corresponding muscle lengths would be roughly equal in tension (Pinniger et al., 2006; Roots et al., 2007).

In a previous study (Till et al., 2008) we quantified the tension increase during lengthening of the $M$. gastrocnemius (Caput mediale) of the rat, assuming that the muscle consists of a tension-generating component (TGC) in series to an elastic component. Experimental results showed the tension gradient at constant lengthening velocity of the TGC to be independent of muscle length in the range up to optimal muscle length(ascending limb of the tension-length relation); tension traces of constant-velocity contractions at different starting muscle lengths within this length range were strikingly parallel(figure 2). Tension increase with TGC length change in eccentric eonstant velocity contractions starting from different muscle lengths, rat M. gastroenemius (Caput mediale). This example of experimental data is taken from. Lengths are given relative to optimum fibre length $\left(l_{f 0}\right)$ and tension is normalized to cross-sectional area. Differences of starting lengths to optimum TGC length are $-0.45 l_{f 0},-0.33 l_{f 0},-0.19 l_{f 0},-0.13 l_{f 0}, 0.07 l_{f 0}$ and $0 l_{f 0}(1-6)$. Extension velocity of whole muscle was $0.77 l_{f 0} / \mathrm{s}$, extension distance was $0.15 l_{f 0}$. In their straight part, the curves are nearly parallel. Parallelism is striking for curves 1 5, while at the longest starting length (eurve 6) there is a slight deviation from parallelism. A Hill-type model 
was suggested under the usual assumption that tension of the TGC without effects of contraction "history" is the product

$\underline{f_{T G C}=f_{i m}} \underline{r_{v}} r_{l}$

( $f_{\text {im }}$ : maximal isometric tension, $r_{v}$ : factor due to the tension-velocity relation, $f_{i}$ : factor due to the tension length relation of the TGG. Parallelism at different starting lengths was evident for different extension velocities (constant within the series), and a term was included to account for enhancement of tension due to eccentric contraction history. The term compensated for the variable gradient of the tension-length relation, to achieve a constant tension gradient at constant $r_{v}$ at different muscle lengths, in accordance with the experimental results.

Throughout the present paper, the designation "enhanced" will be used to indicate an extension-distance dependent increase in tension which is not due t there was no evidence for a dependence of the tension gradient on extension velocity (latter data not reproduced in the influence of the tension-length relationcurrent paper). A phenomenological model was suggested, with a special term to account for the observed parallelism.

We now discuss the problems arising in attempts to explain the constant tension gradient in eccentric contractions of constant velocity with physiological mechanisms. Considering the events of the sliding filament mechanism which are well accepted to determine the tension-length relation (Gordon et al., 1966; Edman, 2005), an important prediction on filament mechanics is derived. An example model, based on physiological mechanisms, is introduced and tested in simulations. The model can also account for enhancedtension after completion of an eccentric contraction

Throughout the present paper the designations "enhanced" or "enhancement" will be used to indicate an extension-distance dependent increase in tension which is not due to the influence of the tension-length relation.

\section{Influence of the tension-length relation}

Figure 1A shows the approximate shape of the tension-length relation, as it is determined from isometric contractions of short segments of muscle fibres (Gordon et al., 1966; Edman and Reggiani, 1987; Edman, 2005), and as it is thought to be representative for a single half-sarcomere. The filaments of a sarcomere are schematically illustrated in figure 1B. The 
ascending limb consists of two ranges with different gradients (1 and 2). The cause of the gradient of range 1 was originally identified with a compression of the ends of opposite thin filaments against each other and/or an overlap of the contralateral thin filament with the ipsilateral thick filament active zone, the so called "double overlap" (Gordon et al., 1966). The data of Elmubarak and Ranatunga (1984) (whole rat muscle) and Edman (2005) (mouse muscle fibre segments) make the latter cause more probable, which could lead to an inactivation of cross-bridges and/or to a counteracting force, possibly by cross-bridge formation with the contralateral thin filament. In range 2 , the gradient is further increased by a compression of the thick filament, leading to a counteracting spring-force and/or an inactivation of cross-bridges.

In eccentric constant-velocity contractions of a whole skeletal muscle in Till et al. (2008), the tension gradient was constant over a wide length range = figure 2. The experiments were performed with a whole muscle, possibly containing different fibre lengths, and without monitoring of sarcomere lengths. However, the range of parallelism corresponding to isometric tensions from $35 \%$ of maximum to maximum is likely to change the length of some muscle fibres between ranges 1 and 2. Furthermore, it is clear from the whole muscles tension-length relation in figure $2 \mathrm{~B}$ that at least some of the muscles fibres assume lengths corresponding to the plateau and even the start of the descending limb over the range of parallelism. Although in a whole muscle variability of fibre lengths can lead to a superposition of different tension-length curves of muscle fibres, so that the borders of their different parts are not clearly distinguishable, this can not be the reason for the observed constant tension gradient (parallelism) since, as seen in figure 2B, the slope of the tension-length relation of the whole muscle was still non-constant. Thus, the range of parallelism included a part of the whole muscles tension-length relation with variable slope, probably comprising lengths with muscle fibres at ranges 2 and 1 of the ascending limb, at the plateau, and even at the start of the descending limb of the fibres tension length relations.

If the tension increase-gradient in eccentric constant-velocity contractions of a half-sarcomere culd be-were solely caused by the mechanisms responsible for the tension-length relation, and if these mechanisms remain-remained unchanged compared to isometric contractions, the tension gradient would be higher in length range 2 than in length range 1 . In eccentric constant velocity eontractions of a whole skeletal muscle in, however, the tension gradient was 
eonstant over a length range corresponding to isometric forces from 15 of optimal to optimal, i.e. a range including range 1 and most of range 2 . not be constant, as observed experimentally, but would follow the variable slope of the isometric tension-length relation. Although in the experiments of the cited paper additional enhancement of tension in an extension-distance dependent way was shown to occur, it seems unlikely that this effect compensates for the differences in the mechanisms-variable slope of the tensionlength relation in ranges 1 and 2 to achieve a length-independent tension gradient tension gradient independent of starting length. To take more plausible assumptionseompatible with the experimental findings-, compatible with a constant tension gradient in eccentric constant-velocity contractions in ranges 2 and 1 of the ascending limb, we hypothesize that:

1. The extension-distance dependent enhancement of tension during eccentric constant-velocity contractions is independent of half-sarcomere length over the whole range of half-sarcomere lengths covered by the experimental data.

2. The tension-length relation in eccentric contractions is different from that in isometric contractions and has a constant gradient over its whole ascending limb.

To account for assumption 2, we note that compression of the thick filament is confined to range 2 of the ascending limb, while the mechanism underlying range 1 is in effect over the whole ascending limb, probably with the same gradient as in range 1 (dashed line I in figure 1A). So the absence of effects of thick filament compression in eccentric contractions would yield the desired constant gradient of the ascending limb. This would imply that a possible counteracting compression force does not diminish gradually, but gets zero instantaneously at the start of extension - e.g. because the thick filament does not extend again as long as the muscle is activated - and that possibly inactivated cross-bridges in the compressed filament either remain inactivated or all get reactivated at once at the start of decompression. In an eccentric contraction starting from the short end of the ascending limb, a counteracting compression force instantaneously getting zero or instantaneously reactivated cross-bridges would lead to an ascending limb as dashed line I in figure 1A, while cross-bridges remaining inactivated would produce dashed line II, which is parallel to dashed line 1 and starts at tension zero. A mixture of these effects might yield a line in between and parallel to the dashed lines.

To extend the length range of constant extension-distance dependent 
enhancement of tension to include the plateau region tension-gradient in eccentric contractions to include regions outside the ascending limb of the isometric tension-length relation, one could assume that even the mechanism underlying range 1 in figure $1 \mathrm{~A}$ is not effective in eccentric contractionsThis could be due to cross bridges inactivated by "double overlap" remaining inactive even if the double overlap is removed during an eccentric contraction. Such an additional hypothesis, however, is not further pursued here since available data for comparing tension gradients of eccentric contractions within we additionally assumed that parts of the thin filament getting into contact with the thick filament during eccentric contractions are not immediately available for crossbridge formation, but change their state to get available depending on contact time in an exponential manner. This has the effect that at a sufficiently large extension velocity the amount of "available" thin filament (if some was available at all) decreases already at the start of elongation, making the gradient of the effective tension-length relation negative, even at the "ascending limb" of the isometric tension-length relation. Without attempting a physiological explanation for the "delayed availability", it is shown below in simulations that this assumption suffices to extend the range of parallelism into the plateau region those within the ascending limbis searceand the start of the descending limb.

\section{Origin of the extension-distance dependent enhancement of ten- sion}

The three possible explanations of enhanced tension after after a stretch suggested in the literature and mentioned in the introduction were considered as causes of the extension-distance dependent enhancement of tension during during an eccentric contraction on the ascending limb of the tension-length relation. Sarcomere length inhomogeneities were-which is, as we hypthesized above, independent of half-sarcomere length over the whole range of half-sarcomere lengths covered by the experimental data. Rapid lengthening of some half-sarcomeres to lengths at which they generate only passive tension ("sarcomere popping", Morgan et al. (2000)) was ruled out since they are it is not believed to enhance tension-take place on the ascending limb (Morgan et al., 2000; Herzog, 2005), and at least at the shorter muscle lengths of our experimental data all half-sarcomeres can be expected to be on the ascending limb. Similarly heterogeneity of half-sarcomere lengthening velocity (Telley et al., 2006) is unlikely as a cause since with 
all half-sarcomeres on the ascending limb any slowing down of extension of an individual half-sarcomere relative to the others will decrease this half-sarcomeres tension relative to the others and thus counteract the slow-down. The extent of any effects of altered cross-bridge kinetics on tension is likely to be proportional to the currently available number of active cross-bridges. Thus, sinee-However, this number is not constant over any length rangeon probably not constant either in range 1 of the ascending limb (the assumed double overlap will inactivate cross-bridges or introduce cross-bridges acting on the "wrong" thin filament) or in range 2 (in the compressed part of the thick filament cross-bridges, if not wholly inactivated, are denser spaced and so are likely to have altered overall activity against the binding sites at the thin filament). Thus, cross-bridge kinetics are unlikely as cause of an extensiondistance dependent enhancement of tension which is constant over a range including the whole ascending limb of the tension-length relation. So we suggest the third possibility, enhanced passive stiffness, as the reason for the extension-distance dependent enhancement of tension.

The sarcomere protein titin is known to be associated with passive muscle tension in response to stretch (e.g. Magid and Law, 1985; Higuchi, 1992; Wang et al., 1993). It is a giant protein $(\approx 3.5 \mathrm{MDa})$, tightly bound to the thick filament and freely extending through the so called I-band of the sarcomere to the Z-disk, where its N-terminal part is fixed (see e.g. Tskhovrebova and Trinick (2002) for a review). The stiffness of I-band titin could be enhanced by an elevated calcium concentration during activation (Labeit et al., 2003). Also, titin stiffness could be indirectly enhanced in activated muscles by adhesion of parts of I-band titin to the much stiffer thin filament, as suggested e.g. by Pinniger et al. (2006). For the latter suggestion, available evidence is scarce (Kellermayer and Granzier, 1996); there is even evidence that a calcium concentration elevated to still physiological values $(10 \mu \mathrm{M})$ inhibits binding of an I-band titin fragment to a thin filament constituent (Kulke et al., 2001). Nevertheless, we assume that binding of Iband titin to the thin filament at muscle activation is the cause of enhanced passive stiffness, since, as detailed in the next section, this can naturally explain the constancy of the extension-distance dependent enhancement of tension over all half-sarcomere lengths.

Thus, since titin is fixed at the end of the thick filament but must be able to generate tension even in eccentric contractions over length range 2 of the ascending limb (where the thick filament is shortened by compression), a prediction inherent to the above conclusions is that the thick filament, as 
long as the muscle is activated, withstands this tension even in a compressed state, i.e. tension will not lead to a re-elongation of the compressed thick filament. This prediction makes the conclusions of the previous subsection more precise. Except for this indirect reasoning, to our knowledge there is no evidence as yet for such a behavior, nor any known mechanism which could lead to such an internal lengthening-resistance of the compressed thick filament.

\section{Example model of a half-sarcomere}

The above conclusions do not define each model detail. For an example model, we choose that in range 1 of the ascending limb, tension is diminished by overlap of the contralateral thin filament with the ipsilateral thick filament active zone ("double overlap"), which completely abolishes active tension generation in the double overlap zone, but generates no counteracting force. In range 2 of the ascending limb, compression of the thick filament is thought to generate only negligible counteracting spring force, and is assumed to reduce tension generation by inactivation of cross-bridges in such a way that active tension is always proportional to the length $l_{o}$ of the (possibly compressed) free part of the thick filament active zone (i.e. the part which is overlapped by the ipsilateral thin filament, but not overlapped by the contralateral thin filament). These choices are in accordance with Edman (2005), who achieved a good fit to an experimental tension length curve of mouse M. flexor digitorum brevis with equivalent numerical numerically equivalent assumptions (although, differently to the current assumptions, diminution of tension by compression of the thick filament was not related to cross-bridge inactivation, but to a counteracting force). In addition, active tension $f_{a}$ depends on the sliding velocity $v$ between the thin and thick filamentand, on a factor $r_{a c t}$ due to activation with $0 \leq r_{a c t} \leq 1$, and on a factor $r_{a u \text { with }}$ $0<r_{\text {au }}<1$ due to "delayed availability" of the thin filament coming into contact with the thick filament (as discussed in section 2), thus

$$
f_{a}=f_{i m} r_{v}(v) r_{a c t} r_{a v} l_{o} / l_{a}
$$

where $f_{i m}$ is the maximum isometric tension, $r_{v}(v)$ is a factor due to the sliding velocity $v$ with $r_{v}(0)=1$, and $l_{a}$ is the uncompressed length of the thick filament active zone.

To account for compression and decompression, the thick filament active zone is modelled as a bidirectional visco-elastic spring which is practically 
inextensible over the uncompressed length $l_{a}$ and very compliant for compression to below $l_{a}$. Viscous resistance of the spring linearly depends on $r_{\text {act }}$. At high activation (large $r_{a c t}$ ), viscous resistance to lengthening is high, practically not allowing (re-)extension. At low activation ( $\operatorname{small} r_{a c t}$ ), viscous resistance is negligible. For shortening, viscous resistance is always negligible, independently of activation.

A sketch of the model is given in figure 3. Adhesion of the central part $j$ of titin to the thin filament is modelled by assigning a viscous resistance to this part. If $j$ moves away from the Z-disk, resistance linearly depends on $r_{\text {act }}$, being high for full activation and negligibly small if there is no activation. For movement of $j$ towards the Z-disk, we have to account for shortening (concentric) contractions. In shortening, the central part $j$ of titin is supposed to move freely towards the Z-disk, to avoid the generation of counteracting forces by adhesion of $j$ to the thin filament. To achieve this, resistance is assumed to be negligibly small even during activation if $j$ moves towards the Z-disk. Physiological explanations for this could be a drop of calcium concentration during concentric contractions (Vandenboom et al., 1998) or a direct dependence of resistance on the direction of tension.

The distal part of titin is supposed to generate extension-distance dependent passive tension in eccentric contractions and is therefore modelled as a linear spring. The length of the spring at zero tension is assumed to be very short and is set to zero for simplicity. This short force-less length is necessary to let generation of tension start as soon as the eccentric contraction begins, so that no upwards bent in the tension trace is produced some time after the start of extension. Since passive tension of the inactive half-sarcomere is-muscle was negligible in the length range of the ascending timb of the tension-length relation of the activated muscleexperimental data refered to, no attempt is made to realistically account for this passive tension and proximal titin is arbitrarily modelled as a linear spring with negligible tension and a length of zero at zero tension.

Note that the above modelling of adhesion of central titin to the thin filament with a viscous resistance makes passive tension depend not only on extension distance, but also on extension velocity: at higher extension velocity, tension elevation is faster leaving less time for "sliding" of the central titin $\bar{m}$ so tension elevation needs less sarcomere extension, since less central titin "sliding" has to be compensated for. This is in contrast to our experimental data, where we found no evidence for a dependence of the tension gradient on extension velocity. However for large values 
of viscous resistance the velocity-dependence of the tension gradient may be small and not easily evident in experimental data. Also the assumed negative gradient of the effective tension-length relation in contractions of sufficient extension velocity (see assumption of "delayed availability" of the thin filament, section 2), together with the positive slope of the eccentric tension-velocity relation, counteracts this velocity-dependence.

We previously published a model which, among others, detailed the way I-band titin could increase its stiffness in eccentric contractions (Rode et al., 2009). Briefly, an elastic central region of titin (the so called PEVK region) was assumed to attach at multiple binding sites of the thin filament at activation. The single attachments were assumed to withstand a certain disruptive force and to rapidly re-attach after a disruption. Within the physiological length range, this was shown to yield a nearly linear tension-elongation behavior at activation. This is the same effect as achieved by the units $j$ and $d t$ together in the current model (figure 3). However, since the multiple attachments in the previous model do not involve distal titin, a slack distal titin would cause an initial delay of elastic tension development in eccentric contractions at the ascending limb of the tension-length relation. This in turn would lead to an upwards bent in the tension trace some time after the start of extension(not shown), which is not seen in our experimental contractions.

\section{Simulation}

Simulations of eccentric contractions with the example model are performed in order to test whether they are indeed in accordance with experimental results. Since simulations will be qualitatively compared with tension traces of experiments with a whole muscle, the modelled conditions are partially adapted to those within a real muscle by including an elastic component in series to the half-sarcomere into the model. Without this, imposed velocity changes of the whole muscle would be identical to velocity changes of the tension-generating component, which would lead to unrealistically sharp velocity gradients and corresponding tension gradients. The length of this "series elastic component" (SEC) at zero tension is unimportant for our purpose and is set to zero. The development over time of the length $l:=l_{s}+\Delta l_{S E C}$, where $\Delta l_{S E C}$ is the extension of the SEC, is an input of the model. Length is given in units of optimal length $l_{\text {opt }}$ defined as $l_{\text {opt }}:=l_{\text {thin }}+\left(l_{z}-l_{b}\right) / 2$ ( $=$ short end of the plateau $)$. 
Four-Five coupled differential equations were used to describe the development over time of, respectively, the half-sarcomere length $l_{s}$, the distance $l_{p t}$ of $j$ from the middle of the Z-disk, the length $l_{\text {thick }}:=l_{\text {act }}+l_{b} / 2$ of half the thick filament, and the state of activation $r_{a c t}$ and the factor $r_{a x}$ due to "delayed availability" of the thin filament - see figure 3 for explanation of lengths:

$$
\begin{aligned}
\frac{d l_{s}}{d t} & =r_{v}^{-1}\left(\frac{\left(f_{S E C}\left(l-l_{s}\right)-f_{d t}\left(l_{s}-l_{\text {thick }}-l_{p t}\right)\right) l_{a}}{f_{i m} r_{a c t}\left(l_{\text {sovl }}-l_{\text {dovl }}\right)} \frac{\left(f_{S E C}\left(l-l_{s}\right)-f_{d t}\left(l_{s}-l_{\text {thick }}-l_{p t}\right)\right) l_{a}}{f_{i m} r_{a c t} f_{a v}\left(l_{\text {sovl }}-l_{d o v l}\right)}\right) \\
\frac{d l_{p t}}{d t} & =v_{j}\left(f_{d t}\left(l_{s}-l_{\text {thick }}-l_{p t}\right)-f_{p t}\left(l_{p t}\right), r_{a c t}\right) \\
\frac{d l_{\text {thick }}}{d t} & =v_{t}\left(f_{d t}\left(l_{s}-l_{\text {thick }}-l_{p t}\right)-f_{\text {thick }}\left(l_{\text {thick }}\right), r_{a c t}\right) \\
\frac{d r_{a c t}}{d t} & =\left(S-r_{a c t}(t)\right) / k_{a c t} \\
\frac{d r_{a v}}{d t} & \equiv \frac{\partial r_{a v}}{\partial t}+\frac{\partial r_{a v}}{\partial b_{l}} \frac{d b_{l}}{d t}+\frac{\partial r_{a v}}{\partial b_{u}} \frac{d b_{u}}{d t} .
\end{aligned}
$$

In the first equation, $r_{v}^{-1}$ is the reverse function of $r_{v}\left(f_{a}\right)$ (tension-velocity relation), $l_{\text {sovl }}=\min \left\{l_{\text {thin }}+l_{\text {thick }}+l_{z} / 2-l_{s}, l_{\text {thick }}-l_{b} / 2\right\}$ is the length of single overlap, and $l_{\text {dovl }}=\max \left\{0, l_{\text {thin }}+l_{z} / 2-l_{s}-l_{b} / 2\right\}$ is the length of double overlap. In the fourth equation, which models activation as an exponential function of time, during stimulation $S=1$ and $k_{a c t}=k_{o n}$, while after stimulation $S=0$ and $k_{a c t}=k_{o f f}$. The fifth equation is the result of a restriction of the model to simplify simulation: i) Eccentric contractions must start with $r_{a u} \approx 1$ (sufficiently long isometric pre-contraction). ii) The current lower border of the contact range of thin and thick filament must never be larger than the initial upper border of that range. The compliance with these restrictions has been checked in the result of each simulation. Also, the fact is used that due to the assumed exponential time-dependence, the change over time of mean availability in a constant length-interval of thin-thick filament contact in activated muscle is proportional to the difference of this mean availability to one. $\partial r_{a x} / \partial t$, the derivative of $r_{a y}$ over time in the current length-interval of thin-thick filament contact, is calculated as a linear function of $r_{\text {act }}$ ranging from $-r_{a x} k_{\text {ua }}$ for $r_{\text {act }}=0$ to $\left(r_{\text {au }}-1\right) * k_{a u}$ for $r_{\text {act }}=1$. $\partial r_{a u} / \partial b_{l}$ and $\partial r_{a v} / \partial b_{u}$ are the changes of $r_{a u}$ due to changes of the lower $\left(b_{l}\right)$ and upper $\left(b_{u}\right)$ border of the contact interval and are easily calculable in eccentric contractions (due to the above model restrictions) from current $r_{a w}$ and interval length; the velocities $d b_{l} / d t$ and $d b_{u} / d t$ are calculated from $d l_{s} d t$ and the filament lengths. 
The functions $r_{v}, f_{S E C}$ (tension of SEC), $f_{d t}$ (tension of distal titin), $v_{j}$ (velocity of $j$ ), $f_{p t}$ (tension of proximal titin), $v_{t}$ (extension velocity of thick filament active zone), and $f_{\text {thick }}$ (tension of modelled spring in thick filament active zone) are specified as follows:

The tension-velocity relation is defined according to Hill (1938) and van Soest and Bobbert (1993) as

$$
r_{v}(v)= \begin{cases}\frac{a_{r} v+b}{b-v} & \text { if } \quad v \leq 0 \\ \frac{B(1-A)}{B-v}+A & \text { if } \quad v>0\end{cases}
$$

where $a_{r}$ is defined as $a / f_{i m}$, and $a$ and $b$ are parameters of Hills equation; $B$ is defined as $b(1-A) /\left(\kappa\left(a_{r}+1\right)\right)$ and parameters $A$ and $\kappa$ are a tension limit relative to $f_{i m}$ and the slope change of the tension-velocity curve at $v=0$, respectively. $f_{S E C}$ is modelled according to Winters (1990):

$$
f_{S E C}\left(\Delta l_{S E C}\right)= \begin{cases}\frac{f_{S E C 1}}{e^{k_{S h}-1}}\left(e^{\frac{k_{s h} \Delta l_{S E C}}{d_{S E C 1}}}-1\right) & \text { if } \Delta l_{S E C} \leq d_{S E C 1} \\ f_{S E C 1}+s\left(\Delta l_{S E C}-d_{S E C 1}\right) & \text { if } \Delta l_{S E C}>d_{S E C 1} \\ 0 & \text { if } \Delta l_{S E C}<0\end{cases}
$$

where parameters $d_{S E C 1}, f_{S E C 1}$, and $k_{s h}$ are the SEC elongation and the corresponding tension at which the relation changes from exponential to linear, and a shape factor, respectively. $s$ is the slope at elongation $d_{S E C 1}$ and can be calculated from the three parameters. Distal and proximal titin are modelled as linear springs of length zero at zero tension with coefficients of stiffness $k_{d t}$ and $k_{p t}$, respectively. The bidirectional spring of the thick filament active zone was modelled with the functional equation

$$
f_{\text {thick }}\left(l_{\text {thick }}\right)= \begin{cases}k_{\text {lth }}\left(l_{\text {thick }}-l_{0 t h}\right) & \text { if } \quad l_{\text {thick }} \geq l_{0 t h}, \\ k_{\text {sth }}\left(l_{\text {thick }}-l_{0 t h}\right) & \text { if } \quad l_{\text {thick }}<l_{0 t h},\end{cases}
$$

where $k_{l t h}, k_{s t h}$, and $l_{0 t h}$ are the coefficients of stiffness for lengthening and shortening and the length at zero tension, respectively. For the velocities $v_{j}$ and $v_{t}$ the equations

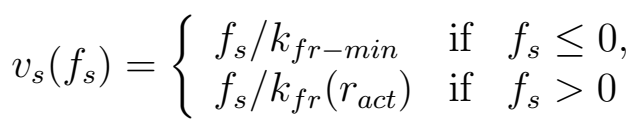

and

$$
k_{f r}\left(r_{a c t}\right)=k_{f r-\min }+r_{a c t}\left(k_{f r-\max }-k_{f r-\min }\right)
$$


were used with $v_{s}=v_{j}$ or $v_{s}=v_{t}$ and suitable values for $k_{f r-\min }$ and $k_{f r-\max }$, which are the minimal and maximal coefficient of friction, respectively.

Filament related lengths (see figure 3) are taken from : $l_{\text {thin }}=1.10 \mathrm{\mu m}, l_{a}$ (defined by $l_{a c t}$ in the uncompressed state of act) $=0.73 \mu \mathrm{m}, l_{b}=0.16 \mu \mathrm{m}, l_{z}$ $=0.05 \mathrm{~mm}$, and are normalized to optimal half-sarcomere length $l_{o p t}:=l_{\text {thin }}+\left(l_{b}+l_{z}\right) / 2$. The parameter $l_{\text {oth }}$ (half thick filament length at zero spring force) is defined by $l_{a}+l_{b} / 2$. The parameters of equations 3 and 4 are those experimentally determined in for rat $M$. gastrocnemius (Caput mediale) (rat no. 4), normalized to $l_{\text {opt }}$ and $f_{i m}: a_{r}=0.26, b=2.26 s^{-1}, A=1.59, \kappa=3.0, k_{s h}=1.79, d_{\text {SEC1 }}$ $=0.56, f_{S E C 1}=0.4$. The remaining parameters are arbitrarily set: $k_{o n}=$ $0.0001, k_{o f f}=0.001 ; k_{d t}=3$ (distal titin); $k_{p t}=0.01$ (proximal titin); $k_{l t h}=$ $10.000, k_{s t h}=0.1$ (thick filament active zone); $k_{f r-m i n}=0.00015, k_{f r-m a x}=$ 150 (junction between proximal and distal titin); $k_{f r-m i n}=0.0001 \mathrm{~s}, k_{f r-\max }$ $=10.000$ s (thick filament active zone).

The model parameters used in simulation are given in table 1. Equations 2 are numerically solved with the 'LSODE' software (Hindmarsh, 1980) distributed with the numerical computing language Octave, whose algorithm uses the backward differentiation formula and adaptive time stepping. The initial value values of $r_{a c t}$ is and $r_{a v}$ are zero. For $l_{s}, l_{p t}$, and $l_{\text {thick}}$, initial values are iteratively solved for using the condition $f_{S E C}=f_{p t}=f_{d t}=f_{\text {thick }}$, or are calculated from the sums of the respective spring lengths if tension is zero.

Tension traces of simulated eccentric constant-velocity contractions at half-sarcomere lengths up to optimal-(figure 4) show the typical time course of experimental eccentric contractions: a first phase with a higher slope, followed by a second phase with an almost linear tension increase, corresponding to a constant extension velocity of the tension-generating component (Till et al., 2008), which in the case of the current model corresponds to the half-sarcomere. As intended by model design, the traces are parallel in their last phase over all parts of the isometric tension-length relation up to the start of its descending limb. Note that no attempt was made to adjust model parameters to achieve tension traces exactly identical to those of the experimental data (figure 2). The reasons are that as discussed in Till et al. (2008), the non-straight first part of the experimental traces is influenced by an inaccurate determination of SEC elongation, and in the straight part of the experimental traces the absolute value of the tension gradient is likely to be additionally influenced by fatigue, which is not covered by the current model. Figure 5 shows a simulation which in- 
cludes an isometric phase immediately after an eccentric contraction. In this phase, tension is still elevated. This means that the current model ean also explain "force enhancement" after completion of a stretchseen to decay towards the purely isometric tension, due to a decay in elastic tension of distal titin. Note that simulation of "delayed availability" of the thin filament (see section 2) obviously does not much affect the late phase of overall tension decay since decaying "extra tension" is roughly equal to the plotted distal-titin tension. The decay in distal-titin tension can be attributed to a "sliding" of the titin junction $j$ along the thin filament. Thus, long lasting or "permanent" residual force enhancement is not present. This is in accordance with experimental results of Julian and Morgan (1979) and Edman et al. (1982) with single muscle fibres at lengths shorter than the descending limb of the tension-length relation. To achieve long lasting force enhancement, as described for fibre lengths at the descending limb (and even at shorter fibre lengths $=$ Peterson et al. (2004)) elastic forces in the proximal titin at longer half-sarcomere lengths could slow down the sliding of the titin junction $j$. However additional assumptions would be necessary to explain "permanent" force enhancement. According to this view, the half-sarcomere length beyond which residual force enhancement is present is not caused by characteristics of the active tension-length relation. In the current paper, elastic tension in the proximal titin at longer half-sarcomere lengths has not been attempted to be modeled realistically.

\section{Summary}

Previous experimental results of a length-independent increase of tension the independence on starting length of the tension increase with extension distance in eccentric constant-velocity contractions, together with well accepted assumptions of the sliding filament theory, restrict the possibilities to suggest a suitable mechanism underlying the tension increase during eccentric contractions. It is plausible to assume that the extension-distance dependent enhancement of tension (defined as the component of extension-distance dependent tension increase not explainable by the tension-length relation) is constant at each half-sarcomere length and that the tension-length relation in eccentric contractions is different compared to that in isometric contractionsand has a constant gradient over its whole ascending limb. Furthermore, the constancy of the extension-distance dependent enhancement of tension favors the hypothesis that enhanced titin-stiffness during activation is its cause. 
These assumptions in turn lead to the prediction that the thick filament retains a shortened length in eccentric contractions starting at half-sarcomere lengths with thick filament compression, even though tension of titin acts to re-extend the thick filament. It is worth noting that this prediction probably can be checked experimentally, since there are fixation techniques which can preserve the state of filaments during a contraction for electron-microscopical examination (Sosa et al., 1994). A further assumption, delayed availability of thin filament in contact with the thick filament, is introduced for compliance with the wide length range of available experimental data on independence of the tension gradient on starting length. An example model is implemented and checked by simulations. Note that we have no information as yet on differences of the derived mechanism between different fibre types of skeletal muscle.

\section{Acknowledgments}

This work was supported by grants no. SI 841/2-2 and SI 841/2-3 of the DFG (Deutsche Forschungsgemeinschaft, Germany).

\section{References}

Abbott, B. C., Aubert, X. M., May 1952. The force exerted by active striated muscle during and after change of length. J Physiol 117 (1), 77-86.

Edman, K. A., Elzinga, G., Noble, M. I., Nov 1982. Residual force enhancement after stretch of contracting frog single muscle fibers. J Gen Physiol $80(5), 769-784$.

Edman, K. A., Reggiani, C., Apr 1987. The sarcomere length-tension relation determined in short segments of intact muscle fibres of the frog. J Physiol $385,709-732$.

Edman, K. A. P., May 2005. Contractile properties of mouse single muscle fibers, a comparison with amphibian muscle fibers. J Exp Biol 208 (Pt 10), 1905-1913.

Elmubarak, M. H., Ranatunga, K. W., May 1984. Temperature sensitivity of tension development in a fast-twitch muscle of the rat. Muscle Nerve 7 (4), 298-303. 
Gordon, A. M., Huxley, A. F., Julian, F. J., May 1966. The variation in isometric tension with sarcomere length in vertebrate muscle fibres. J Physiol 184 (1), 170-192.

Herzog, W., Mar 2005. Force enhancement following stretch of activated muscle: critical review and proposal for mechanisms. Med Biol Eng Comput 43 (2), 173-180.

Higuchi, H., Mar 1992. Changes in contractile properties with selective digestion of connectin (titin) in skinned fibers of frog skeletal muscle. J Biochem 111 (3), 291-295.

Hill, A. V., 1938. The heat of shortening and the dynamic constants of muscle. Proc. R. Soc. Lond. B Biol. Sci. 126, 136-195.

Hindmarsh, A. C., 1980. Two new initial value ordinary differential equation solvers. ACM SIGNUM Newsletter 15, 10-11.

Julian, F. J., Morgan, D. L., Aug 1979. The effect on tension of non-uniform distribution of length changes applied to frog muscle fibres. J Physiol 293, 379-392.

Kellermayer, M. S., Granzier, H. L., 1996. Calcium-dependent inhibition of in vitro thin-filament motility by native titin. FEBS Lett. 380, 281-286.

Kulke, M., Fujita-Becker, S., Rostkova, E., Neagoe, C., Labeit, D., Manstein, D. J., Gautel, M., Linke, W. A., Nov 2001. Interaction between PEVKtitin and actin filaments: origin of a viscous force component in cardiac myofibrils. Circ Res 89 (10), 874-881.

Labeit, D., Watanabe, K., Witt, C., Fujita, H., Wu, Y., Lahmers, S., Funck, T., Labeit, S., Granzier, H., Nov 2003. Calcium-dependent molecular spring elements in the giant protein titin. Proc Natl Acad Sci U S A 100 (23), 13716-13721.

Magid, A., Law, D. J., Dec 1985. Myofibrils bear most of the resting tension in frog skeletal muscle. Science 230 (4731), 1280-1282.

Mehta, A., Herzog, W., 2008. Cross-bridge induced force enhancement? J. Biomech. 41, 1611-1615. 
Morgan, D. L., Whitehead, N. P., Wise, A. K., Gregory, J. E., Proske, U., 2000. Tension changes in the cat soleus muscle following slow stretch or shortening of the contracting muscle. J. Physiol. 522, 503-513.

Peterson, D. R., Rassier, D. E., Herzog, W., Jul 2004. Force enhancement in single skeletal muscle fibres on the ascending limb of the force-length relationship. J Exp Biol 207 (Pt 16), 2787-2791.

Pinniger, G. J., Ranatunga, K. W., Offer, G. W., 2006. Crossbridge and non-crossbridge contributions to tension in lengthening rat muscle: forceinduced reversal of the power stroke. J. Physiol. 573, 627-643.

Rode, C., Siebert, T., Blickhan, R., 2009. Titin-induced force enhancement and force depression: A "sticky spring" mechanism in muscle contractions? J. Theor. Biol. 259, 350-360.

Roots, H., Offer, G. W., Ranatunga, K. W., 2007. Comparison of the tension responses to ramp shortening and lengthening in intact mammalian muscle fibres: crossbridge and non-crossbridge contributions. J. Muscle Res. Cell Motil. 28, 123-139.

Sosa, H., Popp, D., Ouyang, G., Huxley, H. E., Jul 1994. Ultrastructure of skeletal muscle fibers studied by a plunge quick freezing method: myofilament lengths. Biophys J 67 (1), 283-292.

Telley, I. A., Denoth, J., Ranatunga, K. W., 2003. Inter-sarcomere dynamics in muscle fibres. A neglected subject? Adv Exp Med Biol 538, 481-500; discussion 500 .

Telley, I. A., Stehle, R., Ranatunga, K. W., Pfitzer, G., Stüssi, E., Denoth, J., May 2006. Dynamic behaviour of half-sarcomeres during and after stretch in activated rabbit psoas myofibrils: sarcomere asymmetry but no 'sarcomere popping'. J Physiol 573 (Pt 1), 173-185.

Till, O., Siebert, T., Rode, C., Blickhan, R., 2008. Characterization of isovelocity extension of activated muscle: A Hill-type model for eccentric contractions and a method for parameter determination. J. Theor. Biol. 255, 176-187.

Tskhovrebova, L., Trinick, J., Feb 2002. Role of titin in vertebrate striated muscle. Philos Trans R Soc Lond B Biol Sci 357 (1418), 199-206. 
van Soest, A. J., Bobbert, M. F., 1993. The contribution of muscle properties in the control of explosive movements. Biol. Cybern. 69, 195-204.

Vandenboom, R., Claflin, D. R., Julian, F. J., Aug 1998. Effects of rapid shortening on rate of force regeneration and myoplasmic $[\mathrm{Ca} 2+]$ in intact frog skeletal muscle fibres. J Physiol 511 ( Pt 1), 171-180.

Wang, K., McCarter, R., Wright, J., Beverly, J., Ramirez-Mitchell, R., Apr 1993. Viscoelasticity of the sarcomere matrix of skeletal muscles. The titinmyosin composite filament is a dual-stage molecular spring. Biophys J 64 (4), 1161-1177.

Winters, J. M., 1990. Hill-based muscle models: a systems engineering perspective. In: Winters, J. M., Woo, S. L.-Y. (Eds.), Multiple muscle systems, Biomechanics and movement organization. Springer-Verlag New York Inc. 

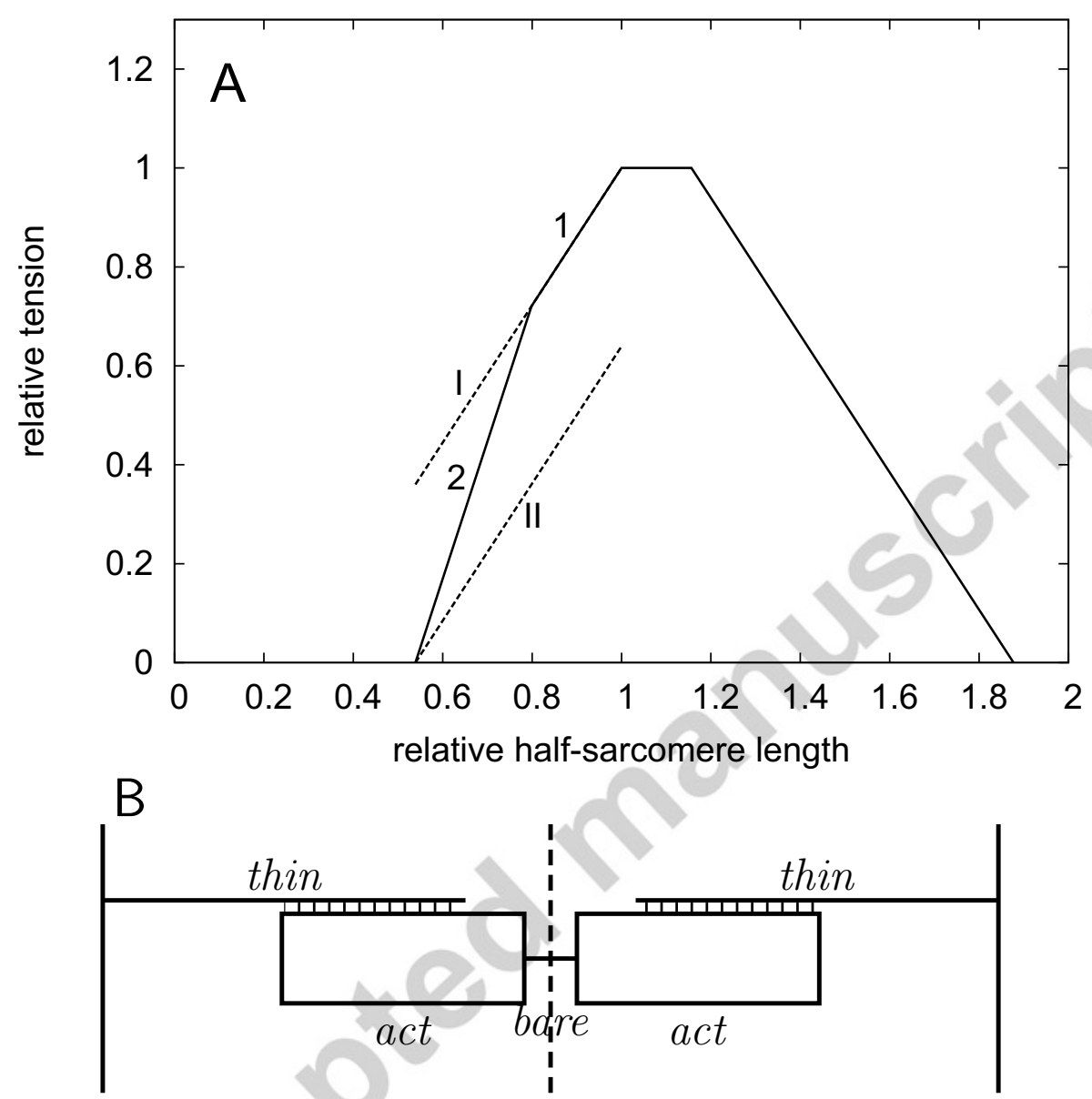

Figure 1: A: Approximate shape of the tension-length relation of a half-sarcomere. Diminution of tension at half-sarcomere lengths greater than optimal (descending limb) is thought to result from diminished overlap of the thin and thick filaments. For lengths shorter than optimal (ascending limb), two ranges with different gradients are marked 1 and 2. These and the dashed lines I and II are explained in the text. B: Scheme of the interaction of thin and thick filaments within a sarcomere. The dashed vertical line separates the two half-sarcomeres. The solid vertical lines represent the so called Z-disks at the sarcomere ends. thin: thin filaments; act: active zones of the thick filament, forming cross-bridges with the thin filament in the zone of filament contact, where active tension is generated; bare: bare zone of the thick filament, unable to form cross-bridges. 

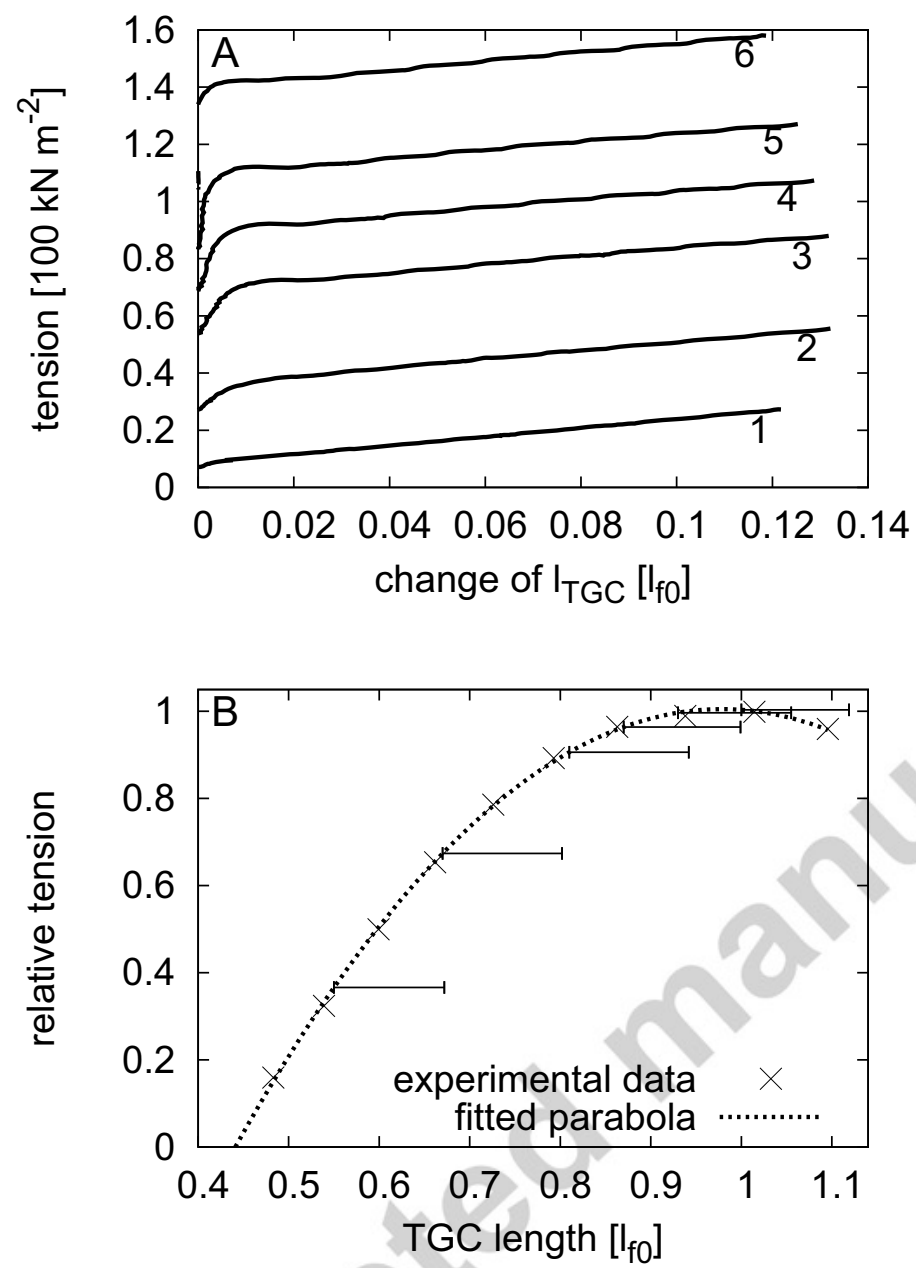

Figure 2: Tension increase with TGC length change in ecentric constant velocity eontractions starting from different muscle lengths, rat $M$. gestrocnemius (Caput mediale). This example of experimental data is taken from. Lengths are given relative to optimum fibre length $\left(l_{f 0}\right)$ and tension is normalized to cross-sectional area. Differences of starting lengths to optimum TGC length are $-0.45 l_{f 0},-0.33 l_{f 0},-0.19 l_{f 0},-0.13 l_{f 0},-0.07 l_{f 0}$, and $\theta l_{f 0}(1-6)$. Extension velocity of whole muscle was $0.77 l_{f 0} / \mathrm{s}$, extension distance was $0.15 \%$. In their straight part, the curves are nearly parallel. Parallelism is striking for curves 1-5, while at the longest starting length (curve 6) there is a slight deviation from parallelism.A: Tension increase with TGC length change in eccentric constant velocity contractions starting from different muscle lengths, rat M. gastrocnemius (Caput mediale). This example of experimental data is taken from Till et al. (2008). Lengths are given relative to optimum fibre length $\left(l_{f 0}\right)$ and tension is normalized to cross-sectional area. Extension velocity of whole muscle was $0.77 l_{f 0} / \mathrm{s}$, extension distance was $0.15 l_{f 0}$. Traces are numbered in the order of increasing starting length. In their straight part the curves are nearly parallel. Parallelism is striking for curves 1-5, while at the longest starting length (curve 6) there is a slight deviation from parallelism. B: Length ranges (horizontal bars) of the contractions in A with respect totohe muscles isometric tension-length relation. Differences of starting lengths to optimum TGC length are $-0.45 l_{f 0}-0.33 l_{f 0}-0.19 l_{f 0}$, $=0.13 l_{f_{0}}-0.07 l_{f 0}$ and $0 l_{f 0}(1-6$ in A). 


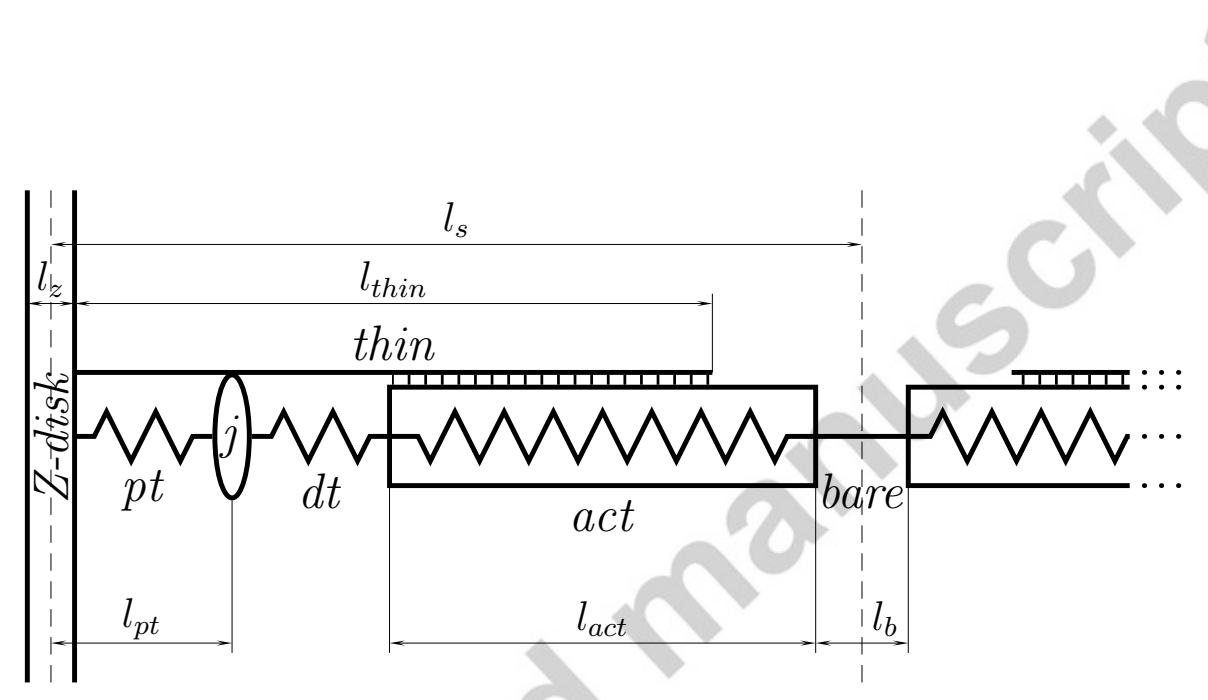

Figure 3: Example model of a half-sarcomere. The dashed vertical lines are the borders of the half-sarcomere. thin: thin filament; act: active zone of the thick filament; bare: bare zone of the thick filament; $p t$ : proximal titin; $d t$ : distal titin; $j$ : junction between proximal and distal titin, able to adhere to the thin filament. Please see text for explanation. 


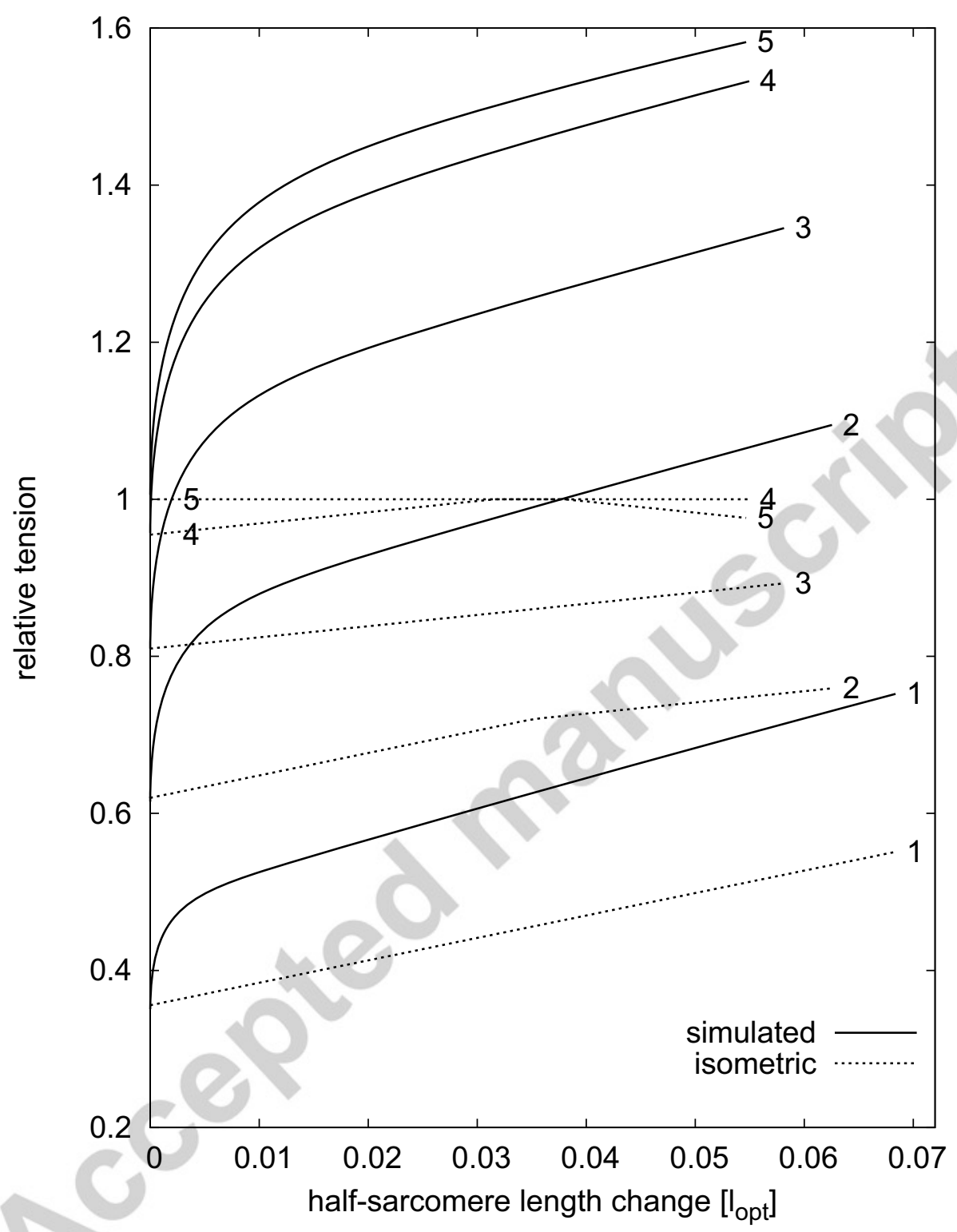

Figure 4: Simulated contractions with constant extension velocity. Tension is given relative to maximum isometric tension. The different starting lengths, relative optimal length $t_{o p t}$ (defined as the border be the ascending limb and the plateat of the tension length relation), are indicated at the traces. Each eccentric contraction starts after an isometric pre-contraction of $0.51 .0 \mathrm{~s}$ (not shown) and covers a length interval of $0.1 l_{\text {opt }}$ at a velocity of $1 l_{\text {opt }} / s$ (length change and velocity, respectively, of whole muscle, including the series elastic component). Traces are numbered in the order of increasing starting length. The dotted lines show the parts of the isometric tension-length relation corresponding to the length intervals of extension; simulations 1 and 3 are confined to range 2 and 1 of the ascending limb, respectively, simulation 2 crosses the border between these ranges, and simulations 4 and 5 cross the lower and upper border of the plateau region, respectively. The last parts of the simulated tension traces are apparently parallel.Parameter values are given in the text. 


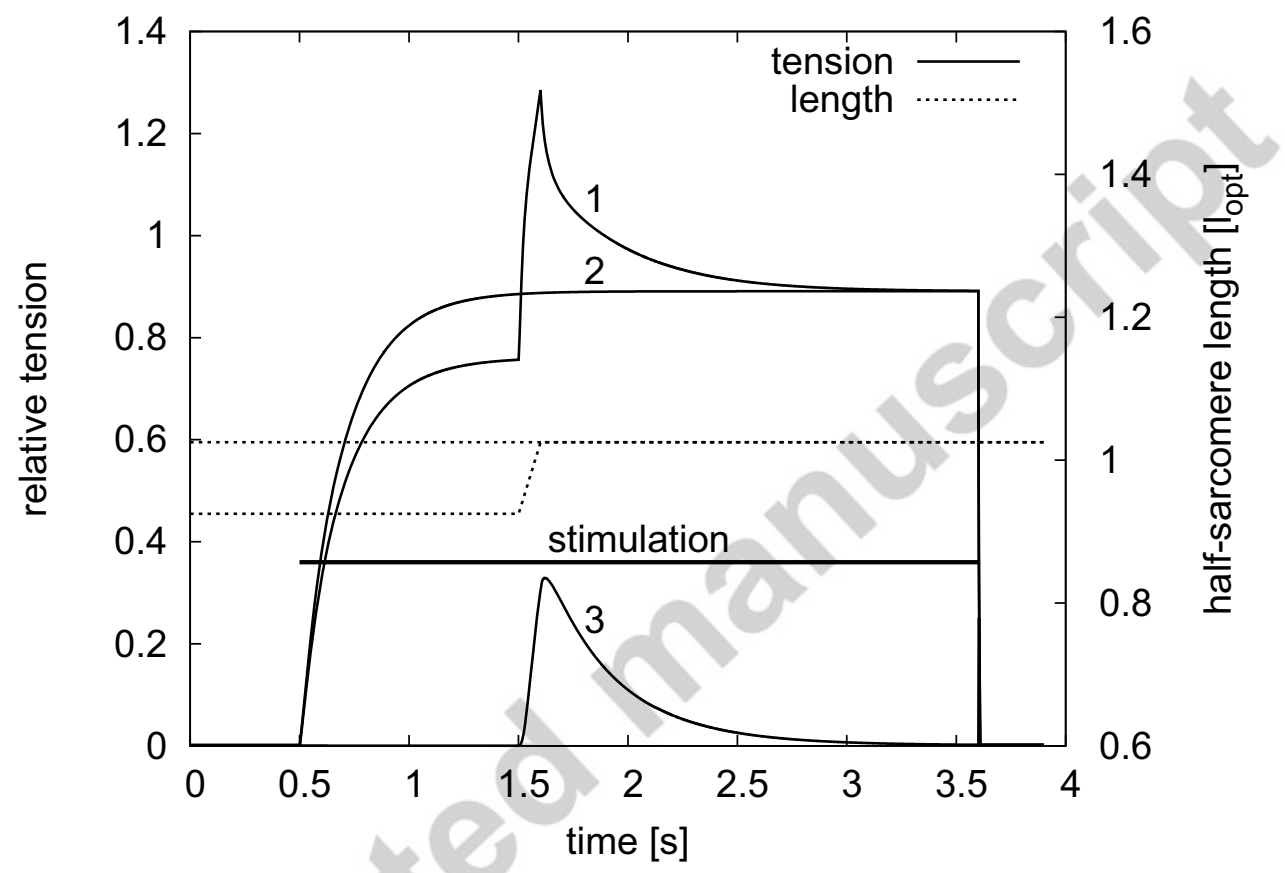

Figure 5: Simulated eccentric contraction with constant extension velocity followed by an isometric contraction, compared to a simulation of a purely isometric contraction at the target length(same parameter values as in figure. Lengths are those of whole muscle, including the series elastic component. Length interval of eccentric contraction: 0.925 to 1.025 4) $l_{\text {opt }}$ 1: tension of eccentric contraction, $2:$ tension of isometric contraction, 3: elastic tension of distal titin in the eccentric contraction. After the end of eccentric movement, the elevated tension decays towards the purely isometric tension. 
Table 1: Model parameters used in simulation.

\begin{tabular}{|c|c|c|c|c|c|c|}
\hline \multicolumn{4}{|c|}{ filament related lengths (see figure 3$)^{\mathrm{a}}$} & \multirow{2}{*}{\multicolumn{3}{|c|}{ tension-velocity relation $(\text { equ. } 3)^{b}$}} \\
\hline \multicolumn{4}{|c|}{$l_{\text {thina }} \quad l_{a \text { a }}^{\mathrm{d}} \quad l_{b_{n}} \quad l_{z_{n}}$} & & & \\
\hline $1.10 \mu \mathrm{m}$ & $0.73 \mu \mathrm{m}$ & $0.16 \mu \mathrm{m}$ & $0.05 \mu \mathrm{m}$ & 2.26 & 1.59 & 3.0 \\
\hline \multicolumn{2}{|c|}{ SEC (equ. 4) ${ }^{b}$} & \multicolumn{3}{|c|}{ activation, availability (equ. 2$)^{\mathrm{c}}$} & \multirow{2}{*}{\multicolumn{2}{|c|}{$\begin{array}{l}\text { distal and proximal titin } \\
k_{d t} \quad k_{n h}\end{array}$}} \\
\hline$k_{s h n} \quad d$ & EC1 $f_{S E C 1}$ & $k_{\text {on }}$ & $k_{a f f \sim}$ & ans $k_{\text {uak }}$ & & \\
\hline 1.79 & 0.4 & 0.0001 & 0.001 & & $\underline{6}$ & 0.01 \\
\hline
\end{tabular}

thick filament (equ. 5) ${ }^{\mathrm{c}} \quad$ equ. 6, 7, titin junction $j$ (left), thick filament (right) ${ }^{\mathrm{c}}$

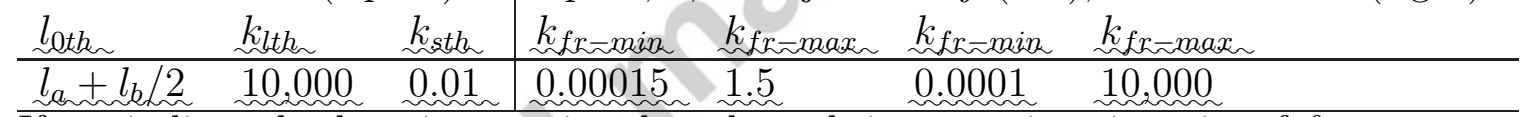
If not indicated otherwise, tension, length, and time are given in units of $f_{\text {imo }}$ $l_{\text {opte and seconds, respectively. }}$

a Lengths according to Edman (2005).

b Parameters from Till et al. (2008), rat $M$. gastrocnemius (Caput mediale) (rat no. 4). Muscle had approximately room temperature $\left(\approx 25^{\circ} \mathrm{C}\right)$. c Parameters arbitrarily set, except $l_{0 t h}$ (half thick filament length at zero spring force, given by $l_{a}$ and $l_{b}$ ). d $l_{a}$ : defined by $l_{\text {act }}$ in the uncompressed state of act. 